Gut, 1988, 29, 254-256

Case report

\title{
Communicating oesophageal duplication: a report of two cases
}

\author{
M L RATAN, R ANAND, S K MITTAL, AND S TANEJA \\ From the Departments of Radiodiagnosis and Paediatric Surgery, Lady Hardinge Medical College and \\ Kalawati Saran Children's Hospital, New Delhi, India
}

SUMMARY Alimentary duplications which include a variety of alimentary cysts, diverticula and tubular malformations are rare abnormalities. Most of them, especially in relation to the oesophagus, do not communicate with the lumen. We present two very rare cases of communicating oesophageal duplication. Both are left sided and unassociated with any other congenital abnormality. These features again are uncommon with such duplications.

Duplications of the alimentary tract include a variety of cysts, diverticula and tubular malformations which are all believed to have a similar embryological origin. These enteric abnormalities may be anywhere from the mouth to the anus and occasionally more than one is present. ${ }^{1}$ In 1944, Ladd and Gross ${ }^{2}$ suggested that tubular or spherical anomalies intimately attached to the alimentary tract should be termed as duplications. The criteria for duplication suggested are: attachment or adherence to some part of the alimentary tract; the presence of a smooth muscle wall, usually in two layers; and an internal lining corresponding to some part of the alimentary tract.

These criteria have helped in simplifying the nomenclature. Certain types of alimentary anomalies which appear to originate from a similar developmental failure would not qualify, however, should these criteria be strictly adhered to. Anderson, Silberman, and Shields ${ }^{3}$ therefore considered as duplications all those alimentary anomalies, either cystic or tubular, surrounded by a muscular covering and intimately related to some part of the alimentary tract.

Although several theories have been offered to explain the origin of the alimentary duplications, the exact mechanism remains obscure. ${ }^{4}$ Among the various explanations for the occurrence of such

Address for correspondence: Dr Rama Anand, A-203 Meera Bagh, Delhi 110041 , India

Received for publication 20 August 1987. anomalies is the diverticulum theory of Lewis and Thyng which states that alimentary duplications are the remains of embryological diverticula that normally regress but may become pinched off to give an adjacent duplication, the vacuolation theory of Sir Arthur Keith which postulates that in the stage of vacuolation of the developing gut some of these vacuoles do not communicate with the definitive gut but remain as isolated units which form cysts or linear vacuoles that coalesce to form a parallel tube, the twinning theory, and the proposal of Black and Benzamin that these so called gastroenteric cysts are the vestigial intrathoracic remains of the vitelline veins. $^{5}$

\section{Case reports}

PATIENT 1

An unknown abandoned male newborn baby was picked up from the road and brought to the hospital by the police. He had mild peripheral cyanosis and marked pulmonary crepitations. Respiratory rate was $52 / \mathrm{min}$. The clinical diagnosis was a tracheooesophageal fistula with aspiration pneumonia. A contrast study of the oesophagus with Dionosil was performed by injecting the contrast through a gastric tube while the tube was being gradually withdrawn. It showed a blind short tubular pouch projecting from the left posterolateral aspect of the upper third of the oesophagus (Fig. 1). The baby died and autopsy confirmed the radiological findings. No other associated congenital anomaly was found. 


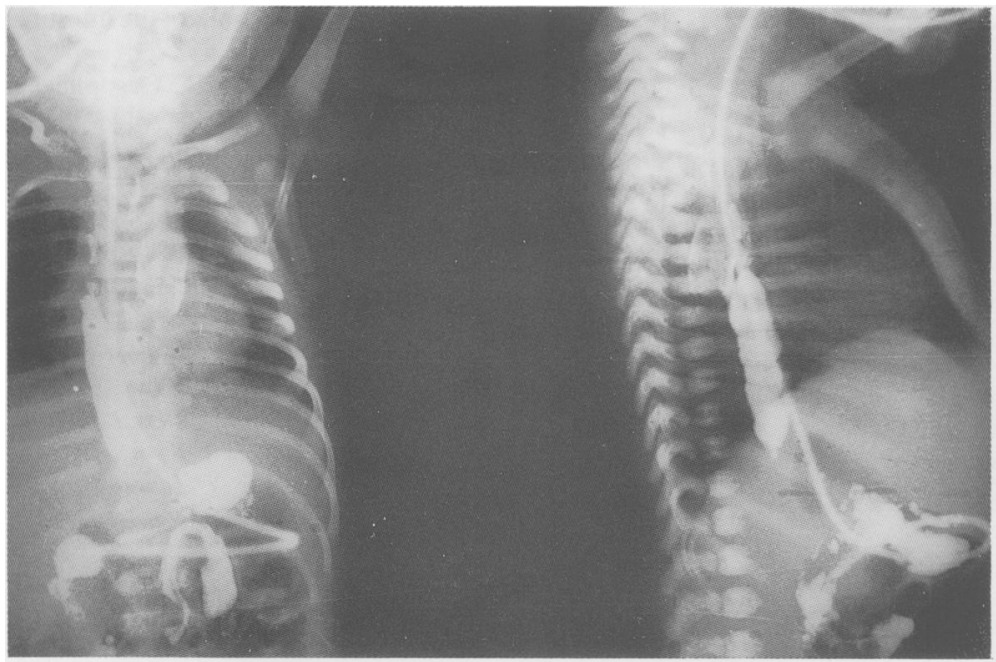

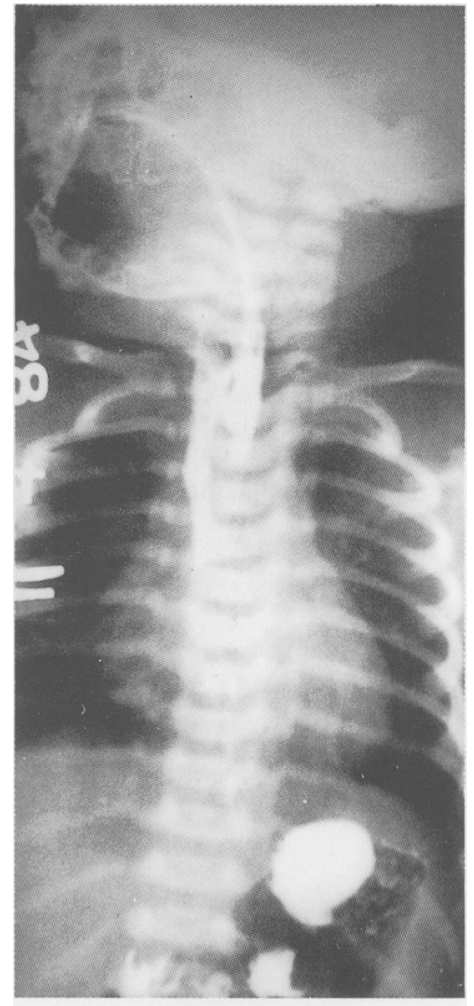

Fig. 2
Fig. 1 Contrast study of the oesophagus (AP and lateral views) showing communicating tubular oesophageal duplication at about the $T_{2-3}$ vertebral level. The gastric tube is in the 'main' oesophagus and stomach.

Fig. 2 Contrast study of the oesophagus (APview) showing communicating tubular oesophageal duplication at about $C_{7}$ vertebral level. The gastric tube is in the tubular duplication.

\section{PATIENT 2}

A one day old boy was born to a primigravida by normal home delivery. The baby had cried several minutes after birth. On admission his general condition was poor, respiration irregular and the respiratory rate was $56 / \mathrm{min}$. He had blood stained frothy secretions from the mouth after feeds. On passing a gastric tube, sometimes the tube would easily pass into the stomach, but at other times it would coil up in the oesophagus. Contrast study of the oesophagus with Dionosil revealed a short tubular blind pouch projecting from the left posterolateral aspect of the upper gullet (Fig. 2), very similar to that seen in patient 1. The child was operated upon and the findings confirmed the radiological findings of a communicating oesophageal duplication. No associated congenital anomaly was found.

\section{Discussion}

Alimentary duplications are rare congenital abnormalities of obscure origin, and usually manifest during the first year of life.' The two cases presented here were both newborn babies. In 1953, Gross" commented on 68 such lesions of which only 16 were thoracic; 13 arose from the oesophagus while three were long tubular diverticula lying within the thorax but arising from the duodenum or jejunum. Eighty per cent of the alimentary duplications do not communicate with the lumen. Oesophageal duplications rarely communicate with the oesophageal lumen, are usually lined by gastric mucosa, they may lie centrally or, more usually, lie to one side, and have been reported twice as commonly and as large on the right as on the left' ${ }^{7}$. Both cases of communicating oesophageal duplication presented here were, however, left sided.

Symptoms vary widely and sometimes the condition may be asymptomatic." Respiratory distress is the commonest manifestation of foregut duplications.' Both the cases presented above had respiratory distress.

Duplications are commonly associated with other congenital malformations, such as spinal deformities, mental retardation, inguinal hernia, club foot, meningocoele, malrotation of the bowel, Meckel's diverticulum, umbilical and diaphragmatic herniae, bilobar right lung, hydrocephalus, congenital heart disease, prematurity and pigeon breast. ${ }^{\times}$There is a high incidence of vertebral anomalies. ${ }^{4}$ In neither of 
the two cases presented here, however, could any associated anomaly be found.

Surgical removal is almost always recommended for the duplication and is usually straightforward, except when inflammation complicates the dissection. ${ }^{10}$

\section{References}

1 Grosfeld JL, O'Neill JA, Clatworthy HW. Enteric duplications in infancy and childhood. Ann Surg 1970; 172: 83-90.

2 Ladd W, Gross S. Abdominal surgery in infancy and childhood. Philadelphia: WB Saunders, 1947.

3 Anderson MC, Silberman WW, Shields TW. Duplications of the alimentary tract in the adult. Arch Surg 1962; 85: 94-108.
4 Bremer JL. Diverticula and duplications of the intestinal tract. Arch Pathol 1944; 38: 132-40.

5 Langston HT, Tuttle WM, Patton TB. Oesophageal duplications. Arch Surg 1950; 61: 949-56.

6 Gross RE. The surgery of infancy and childhood. Philadelphia: WB Saunders, 1953.

7 Borrie J. Duplication of the oesophagus: review and description of 2 cases. Br J Surg 1961; 48: 611-8.

8 Ware GW, Conrad HA. Thoracic duplication of the alimentary tract. Am J Surg 1953; 86: 264.

9 Adkins JC, Kiesewetter. Congenital malformations of the oesophagus. Bluestone $\mathrm{CD}$, Stool SE, eds. Paediatric otolaryngology. Philadelphia: WB Saunders, 1983: 1053-66.

10 Jafek BW, Birney JL, Spofford BT. Congenital oesophageal disorders. ENT Journal 1984; 63: 27-33. 